\title{
Und Aktion! - Konzeptualisierung der Rolle individuellen Akteurshandelns in sozio-technischen Transitionen am Beispiel der regionalen Energiewende im bayerischen Allgäu
}

\author{
Susan Mühlemeier ${ }^{1,2}$ (D) Romano Wyss $^{1,2} \cdot$ Claudia R. Binder $^{1,2}$
}

Zusammenfassung Die deutsche Energiewende gilt als einer der herausforderndsten gesamtgesellschaftlichen Veränderungsprozesse unserer Zeit. Sie zieht einen grundlegenden politischen und ökonomischen Wandel in Deutschland und ganz Europa nach sich. Die fundamentalen Veränderungen, die in Folge der Energiewende stattfinden, sind besonders für Wissenschaftler im Bereich der Forschung zu Nachhaltigkeitstransitionen von Interesse. Die Forschung in diesem Bereich berücksichtigt jedoch bisher die Rolle des individuellen Akteurshandelns für systemische Transitionen noch viel zu wenig. Was treibt Akteure an, die maßgeblich zum Fortschritt der Energiewende in der Region beigetragen haben? Welche Strategien und Aktionsformen wählen sie um die Transition voranzutreiben, wie hängen diese Strategien und Aktionsformen vom sozialen und technischen Umfeld ab, das sie umgibt und wie reagiert dieses Umfeld auf das Handeln der Change Agents? Zur Untersuchung dieser Fragen wenden wir das Human-Environment Systems (HES) Framework (Scholz, 2011) erstmals auf sozio-technische Systeme an. Auf Basis einer exemplarischen Untersuchung des Fallbeispiels der regionalen Energiewende im bayerischen Allgäu schlagen wir eine Anpassung des HES Frameworks für sozio-technische Energiesysteme vor.

Susan Mühlemeier

susan.muehlemeier@epfl.ch

1 Laboratory for Human Environment Relations in Urban Systems (HERUS), Institute of Environmental Engineering (IIE), School of Architecture, Civil and Environmental Engineering (ENAC), École polytechnique fédérale de Lausanne EPFL, Station 2, 1015 Lausanne, Schweiz

2 Lehr- und Forschungseinheit für Mensch-Umwelt-Beziehungen, Department für Geographie, Ludwig-Maximilians-Universität München (LMU), München, Deutschland
Abschließend reflektieren wir, welchen Beitrag das adaptierte HES Framework zur Konzeptualisierung und einem besseren Verständnis von individuellem Akteurshandeln im Kontext sozio-technischer Transitionen leisten kann.

Schlüsselwörter Akteurshandeln · Umfeldwahrnehmung · Motive · Strategien · Energiewende · Allgäu

And Action! - Role of Individual Agency in SocioTechnical Transitions - the Example of a Regional Energy Transition in the Allgäu Region, Bavaria

Abstract The German "Energiewende" is one of the most ambitious societal transition projects in recent times. It causes fundamental political and economic changes in the energy system in Germany and the whole of Europe. These great societal and technological changes of the current energy regime are of particular interest for research on sustainability transitions. In this research strand, however, the role of individual agency for socio-technical transitions is hardly considered. What drives actors who decisively pushed the regional energy transition? Which strategies and types of action do they choose to foster the transition? How do individual strategies and actions depend on the socio-technical system environment and how does the socio-technical system environment react to the individual actions? To analyse these questions, we apply the Human-Environment Systems (HES) Framework (Scholz, 2011) for the first time to sociotechnical systems. Based on an exemplary analysis of the regional energy transition in the Allgäu region in Germany, we propose an adaptation of the HES Framework for sociotechnical systems. Finally, we reflect on how the adapted HES framework for socio-technical systems can contribute 
to a better understanding of individual agency in systemic transitions.

Keywords Human-environmental systems (HES)framework $\cdot$ Socio-technical systems $\cdot$ Multi-level perspective $(\mathrm{MLP}) \cdot$ Sustainability transitions

\section{Einleitung}

Das waren zwei Hand voll Leute, die wollten das und das war für mich aus der heutigen Rückbetrachtung ein ganz seltener Umstand, wie vielleicht zwei Lichter auf dem Schneepflug, die getrennt takten und irgendwann zusammen takten. Das waren hier ein paar Lichter, die haben zusammen getaktet, aus der Landwirtschaft, Forstwirtschaft, Politik und Industrie (Forstwirt und Manager eines Bauunternehmens im bayerischen Allgäu).

Seit der Reaktorkatastrophe von Fukushima 2011 sind in einigen Ländern, vor allem auch in Deutschland, politische Entscheidungen gefallen, die den Weg zur Transition des Energieversorgungssystems klar in Richtung des Atomausstiegs und der Nutzung erneuerbarer Energien weisen. Mittlerweile sind einige Jahre vergangen, in denen auf allen Ebenen in Deutschland versucht wurde, die Veränderung des Energiesystems voranzutreiben: Auf technischer Ebene wurde der Zubau von Wind-, Sonnen-, Biomasse- und Erdenergie verstärkt, Speichertechnologien wurden weiterentwickelt und der Netzausbau vorangetrieben. Auf ökonomischer Ebene entwickelten sich neue Unternehmensformen (z. B. die Aufspaltungen der großen Energiekonzerne wie EON in Tochterfirmen oder die Gründung von Bürgergenossenschaften zur Finanzierung erneuerbarer Energien) und Marktstrukturen veränderten sich. Neben diesen technischen und ökonomischen Komponenten des Wandels rückte aber immer mehr auch die gesellschaftliche Seite der Energiewende in den Vordergrund und die Frage danach, wie sich der gesellschaftliche Umgang mit ökonomischen, technischen und ökologischen Veränderungen gestaltet.

Wissenschaftler, die im Bereich Transformationsforschung tätig sind, arbeiten seit langem an der Untersuchung der Wechselwirkungen zwischen sozio-ökonomischen und technischen Aspekten in systemischen Transformationen (de Haan und Rotmans 2011; Geels 2004; Geels und Kemp 2006; Geels und Schot 2007; Rotmans und Loorbach 2009). Hier werden z.B. neue Steuerungsmechanismen oder sich verändernde Machtstrukturen untersucht, die mit der Transformation einhergehen (Avelino und Rotmans 2009; Binder et al. 2014; Hecher et al. 2016; Loorbach et al. 2008). Bezüglich der Rolle von Akteuren in soziotechnischen Transitionen zog der Vorwurf, die Akteursebe- ne und genauere Konzeptualisierung von Akteurshandeln sei in der Transitionsforschung unterrepräsentiert (Genus und Coles 2008; Smith et al. 2005; Westley et al. 2013), einige Arbeiten zur Konzeptualisierung und Analyse der Akteursebene in sozio-technischen Transitionen nach sich (Avelino et al. 2014; Avelino und Wittmayer 2016; Grin et al. 2011; Heins und Alscher 2013; Riddell et al. 2012; Sommer und Schad 2014; Wittmayer 2016; Wittmayer und Schäpke 2014). Fischer und Newig (2016) geben in ihrer Literaturanalyse dazu einen umfassenden Überblick über die unterschiedlichen Akteurskonzeptualisierungen in der Transitionsforschung und zeigen auf, dass gegenwärtig etwa gleich viele Beiträge einen systemischen wie einen Akteur zentrierten Zugang haben und damit sowohl die Akteursebene, als auch die Systemebene im Transitionsdiskurs ihre Berücksichtigung finden (Fischer und Newig 2016). Doch auch wenn man von einer umfassenderen Berücksichtigung der Akteursebene in sozio-technischen Transitionen sprechen kann, fehlt bisher nach wie vor ein Framework, das sowohl die Konstitution des Akteurshandelns berücksichtigt, das heißt unterschiedliche Einflussfaktoren auf das Akteurshandeln, wie z.B. individuelle Ziele, Wahrnehmung und Lernprozesse als auch seine Einbettung und Interaktion mit dem sozio-technischen System.

Um diese Konzeptualisierung des individuellen Akteurshandelns - eingebettet in ein komplexes systemisches Umfeld - zu ermöglichen, schlagen wir vor, das sog. Human-Environment Systems (HES) Framework (Scholz et al. 2011; Scholz 2011) am Beispiel von Energiesystemen auf sozio-technische Systeme in Transition anzuwenden eine Anwendung des HES-Frameworks auf Energiesysteme wurde bereits von Binder et al. (2013) vorgeschlagen.

Das HES Framework basiert auf Erkenntnissen der Individualpsychologie, Umwelt-Entscheidungsforschung sowie den Systemwissenschaften und baut daher nicht nur auf etablierten Ansätzen zur Konzeptualisierung des individuellen Akteurshandelns auf, sondern auch auf einem profunden Verständnis komplexer Systeme. Das auf sozio-ökologischen Systemen basierte HES Framework kann auf sozio-technische Systeme übertragen werden, da beide Systemkonzeptualisierungen komplexe Systeme zugrunde legen: Gesellschaft und Umwelt, bzw. technologisches Umfeld werden als inhärent vernetzte und interdependente Subsysteme verstanden, deren Koevolution dies Gesamtentwicklung des Systems ausmacht. Menschliches Handeln ist in diese systemische Umgebung eingebettet und evoziert systemisches Feedback, das dann wiederum Ausgangsbedingung für neue Handlungen sind. Arbeiten zu sozio-technische Systemen (Geels 2002) legen dazu Giddens Strukturationstheorie zugrunde (Giddens 1984), das Mensch-Umwelt System folgt dem Prinzip der Komplementarität und verschiedenen Regulationsmechanismen der Subsysteme (Scholz 2011; Scholz und Binder 2004). 
Das HES Framework liefert somit einen wertvollen $\mathrm{Zu}$ gang, wie die Interaktion zwischen Akteurshandeln und systemischem Umfeld auch in sozio-technischen Systemen besser verstanden werden kann.

Dieser Beitrag verfolgt somit folgende Ziele:

- Übertragung des HES Frameworks auf sozio-technische Systeme und Vorschläge zu seiner Adaptation für soziotechnische Systeme.

- Exemplarische Anwendung des HES Frameworks auf individuelles Akteurshandeln in sozio-technischen Energiesystemen am Beispiel der regionalen Energiewende im bayerischen Allgäu.

- Reflexion zum Beitrag des adaptieren HES Frameworks für die Konzeptualisierung individuellen Akteurshandelns in sozio-technischen Systemen in Transition

Zur Bearbeitung dieser Ziele wird in einem ersten theoretischen Abschnitt das Verständnis sozio-technischer Transitionen auf Basis der Multi-Level Perspektive (MLP) (Geels 2002, 2011) zugrunde gelegt und in der Folge die Grundannahmen des HES-Frameworks erläutert. In einem zweiten Schritt übertragen wir das HES Framework auf soziotechnische Systeme und schlagen konzeptionelle Anpassungen vor. Im dritten Schritt wenden wir das angepasste HES Framework auf die Fallstudie der Energiewende im bayerischen Allgäu an. Dazu stellen wir zunächst die Entwicklungen in der Region und unser methodisches Vorgehen vor, bevor wir anschließend exemplarische Ergebnisse zur empirischen Anwendung des HES Frameworks präsentieren. Zuletzt reflektieren wir, wie dieses angepasste Framework z. B. an die MLP anschlussfähig sein kann und diskutieren andere Verwendungsmöglichkeiten und weiteren Forschungsbedarf, um aufzuzeigen, wie das HES Framework in den "transition studies“ aufgenommen werden könnte und einen Beitrag zum besseren Verständnis der Rolle des Akteurshandelns in sozio-technischen Systemen liefern kann.

\section{Theoretischer Hintergrund - Multi-Level Perspective und Human-Environment Systems Framework}

Um das Handeln des Individuums in dieser Untersuchung genauer zu definieren, wird der Begriff der Change Agents verwendet, der in den Wirtschaftswissenschaften bereits seit einiger Zeit für die Rolle von Unternehmern und Firmen in Transitionsprozessen angewandt wird (vgl. z. B. Neffke et al. 2014). Als Change Agents werden im Rahmen dieser Untersuchung jedoch nicht nur Unternehmer verstanden werden, sondern in einem erweiterten Begriffsverständnis alle Akteure, die den Wandel des Energiesystems aktiv vorantreiben (z. B. auch Politiker oder zivilgesellschaftlich Engagierte, vgl. dazu Späth et al. 2007).
Um das Akteurshandeln innerhalb des Transformationsprozesses eines sozio-technischen Systems zu verorten, wird die sog. Multi-Level-Perspektive (MLP) (Geels 2002) als Ausgangspunkt verwendet. Sie hat sich mit einigen Überarbeitungen (Geels 2011) als viel beachteter Analyserahmen für sozio-technische Systeme in Transformation etabliert. Die MLP identifiziert drei Strukturationsebenen, die ein sozio-technisches System prägen: die „Nische“, das „Regime“ und die „Landschaft“. Die MLP erklärt die Transition dieses Systems dadurch, dass ein grundlegender Wandel in der „Landschaft" stattfindet (z. B. der gesamtgesellschaftliche Wertewandel bezüglich der Haltung zur Atomkraft), das bestehende „Regime“ unter Druck setzt (das Regime umfasst z.B. die bisherige politische Regelung zur Energieversorgung oder die gängigen Marktstrukturen) und somit soziale und technische Innovationen aus der "Nische“ in das Regime übernommen werden (z.B. die technische Entwicklung der Photovoltaik-Zellen und das Entwickeln neuer Finanzprodukte zur Finanzierung der Zellen). Die Multi-Level-Perspektive rückt somit die Nischenprozesse (und damit implizit das Handeln von Nischenakteuren) als einen entscheidenden Faktor in den Fokus der Untersuchung und bietet die Möglichkeit, das individuelle Akteurshandeln innerhalb des Transformationsprozesses zu verankern. Da die MLP jedoch - wie oben erläutert - als Analyserahmen für gesellschaftliche Strukturationsprozesse in sozio-technischen Transitionen entwickelt wurde und nicht zur Analyse der Konstitution des einzelnen Akteurshandelns, das die Strukturation voranreibt (oder verhindert), fehlt der MLP eine entsprechende Konzeptualisierung, wie sich Akteurshandeln konstituiert, bzw. wie es mit dem systemischen Umfeld interagiert.

Dazu ziehen wir das Human-Environment Systems (HES) Framework (Scholz et al. 2011; Scholz 2011) aus dem Bereich der Umwelt-Entscheidungsforschung hinzu. Das HES Framework konzeptualisiert als „Prozess-Struktur Modell“" (Scholz und Binder 2003, 2011, S. 453-454) einerseits die Struktur eines Systems (Entitäten und deren Beziehungen) und andererseits humane Entscheidungund Handlungsprozesse, die das System verändern (Scholz und Binder 2011). Humane Handlungsprozesse werden dabei als Ergebnis einer Interaktion zwischen individuellen Zielen, Umweltwahrnehmungen, Strategien und Lernprozessen konzeptualisiert (vgl. Abb. 1). Die erfolgte Handlung erzeugt dann wiederum Reaktionen der Umwelt auf unterschiedlichen räumlichen Skalen und zeitlichen Horizonten (primary und secondary feedbacks), die erneut vom humanen System wahrgenommen werden, einen Lernprozess verursachen und den Handlungsprozess beeinflussen können (vgl. Abb. 1). Das HES Framework lenkt die Aufmerksamkeit der Untersuchung daher explizit auf die Wechselwirkung zwischen individueller Handlung und verschiedener systemischer Ebenen. 
Abb. 1 HES Framework. (Eigene Darstellung, vereinfacht nach Scholz et al. 2011: 455)

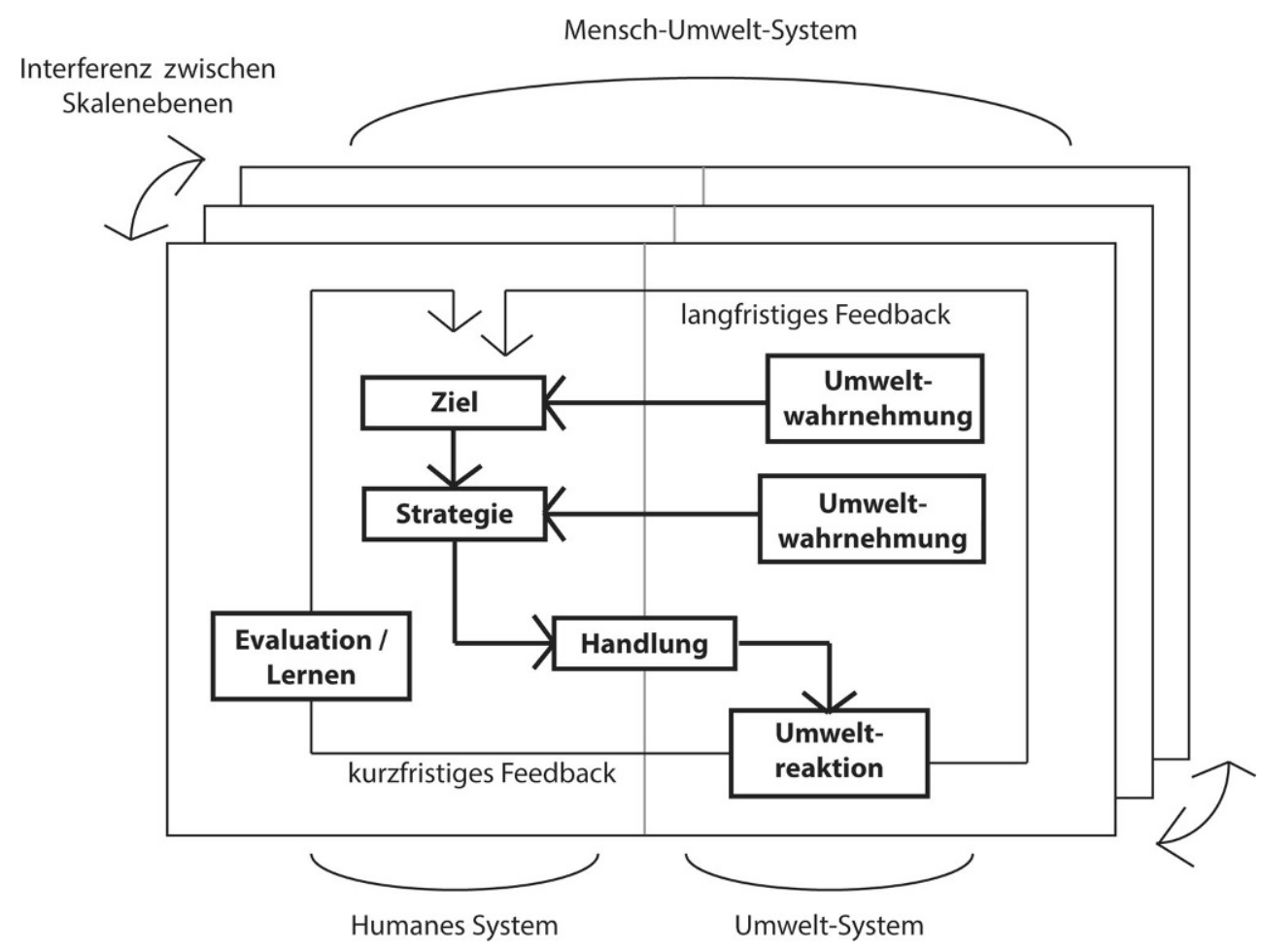

Das HES Framework basiert auf den sieben Postulaten für Mensch-Umwelt Systeme (Scholz 2011), die hier kurz erläutert werden sollen:

- P1 Komplementarität: Mensch-Umwelt Systeme werden als komplexe Systeme verstanden, in denen die humanen und ökologischen Subsysteme miteinander verwoben und voneinander abhängig sind. Das humane Subsystem und das Umweltsystem stellen gleichzeitig jedoch zwei vollkommen verschiedene Systeme dar, die nach unterschiedlichen Regelmechanismen funktionieren (Scholz 2011; Scholz und Binder 2004). Die Verbindung zwischen diesen beiden Subsystemen wird durch die Umweltwahrnehmung des Menschen, sein Handeln und das Feedback der Umwelt auf das Handeln hergestellt (Scholz und Binder 2003).

- P2 Hierarchie: Mensch-Umwelt Systeme lassen sich nach unterschiedlichen Hierarchieprinzipien gliedern (Scholz und Binder 2003, 2004). Regelmechanismen übergeordneter Systeme sorgen dabei für mehr Stabilität als Regelmechanismen untergeordneter Systeme (Scholz und Binder 2003, S. 8) (z. B. Präferenzen des Individuums sind leichter veränderbar und somit weniger stabil als Normen einer Gesellschaft). Scholz und Binder (2003) und Scholz (2011) gliedern das soziale System basierend auf den entsprechenden Regelmechanismen in folgende Levels: Individuum, Gruppe, Organisation, Institution, Gesellschaft und übergesellschaftlich/supranational (Scholz 2011, S. 419; Roland
W. Scholz und Binder 2003, S. 10). Parallel dazu lässt sich auch das Umweltsystem in Hierarchieebenen gliedern, Scholz (2011) schlägt dazu folgende Levels vor: Gemeinschaft, Ökosystem, Landschaft, Ökoregion und Biosphäre (Scholz 2011, S. 414).

- P3 Interferenz: Mensch-Umwelt Systeme beinhalten verschiedene Levels, die sich gegenseitig über die Levelgrenzen aber auch innerhalb eines Levels beeinflussen können. Teilsystem kann somit sowohl über- und untergeordnete als auch gleichgeordnete Systeme beeinflussen (Scholz, 2011, S. 427)

- P4 Rückkopplungen (Feedbacks): Da Mensch-Umweltsysteme als eingebettete Systeme (nested systems) konzeptualisiert werden, entstehen Rückkopplungen (Feedbacks) zwischen diesen Systemen (vgl. Interferenz). Auf Basis des in den Systemwissenschaften grundlegenden Konzept der Hysterese schlägt Scholz (2011) zwei Typen von Rückkopplungen in Mensch-Umweltsystemen vor: Rückkopplungen erster Ordnung, die im direkten zeitlichen Zusammenhang mit einer Aktion stehen sowie Rückkopplungen zweiter Ordnung, die in einem weiteren zeitlichen Abstand zur Aktion stehen (Scholz 2011, S. 432). Für jede Form des Feedbacks gilt, dass Systeme nach einer Handlung in einem physisch veränderten Zustand sind (Scholz und Binder 2004) und Feedbacks grundsätzlich auch über die angesprochenen Levels hinweg passieren (vgl. Interferenz).

- P5 Menschliche Entscheidungen: Menschliche Systeme werden nach Scholz (2011) auf jedem Level von Ent- 
Abb. 2 Das HES Framework adaptiert nach Scholz 2011 (Änderungen grau hinterlegt). (Eigene Darstellung)

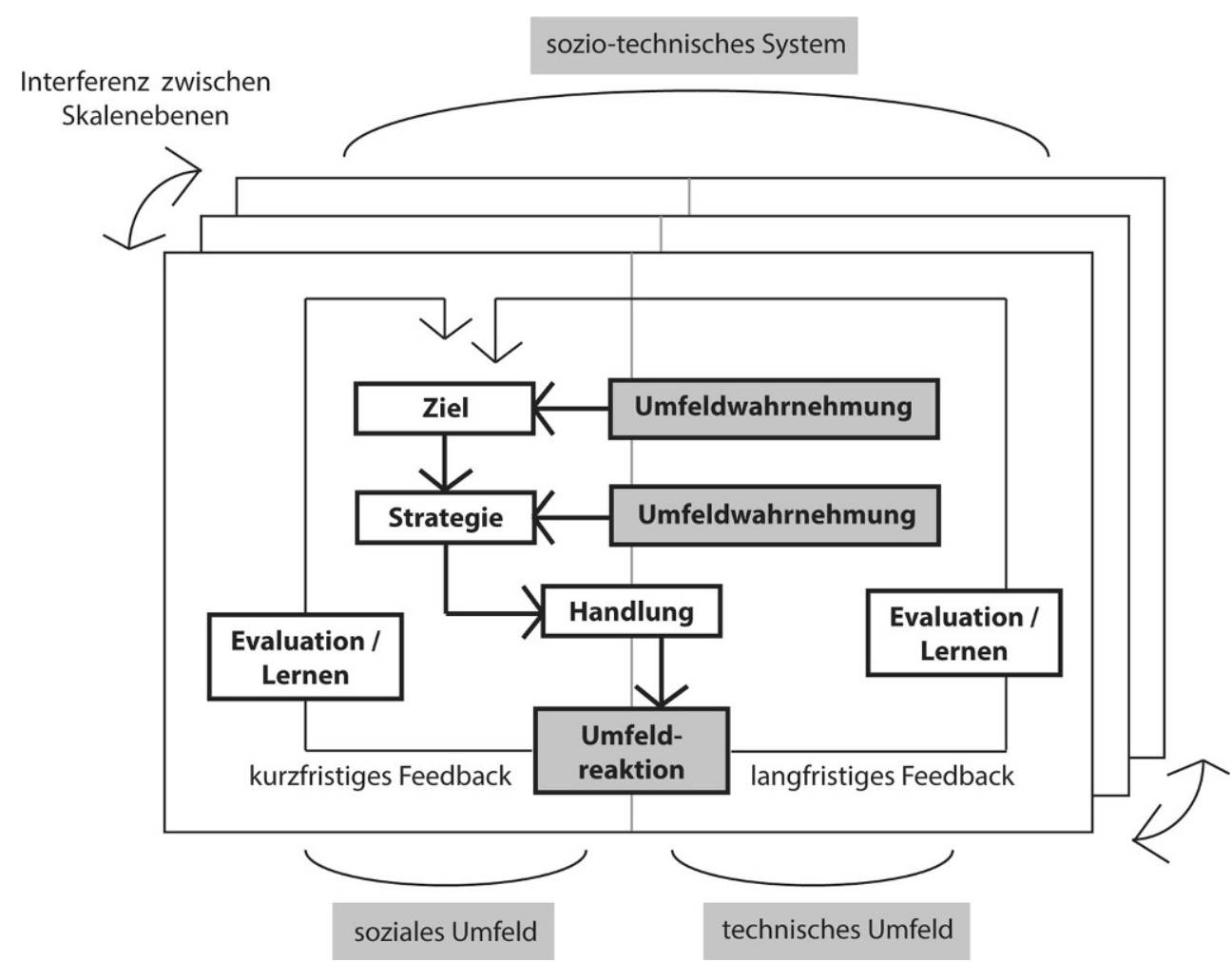

scheidungsprozessen und resultierenden Handlungen geprägt. Entscheidungen sind dabei von individuellen Zielen, der Umweltwahrnehmung und einer bewussten Strategiewahl geprägt. Ziele werden dabei als eher stabile unterliegende Struktur verstanden, die situationsbedingt zur Strategieauswahl aktiviert werden; Strategien stellen eine Art Aktionsplan dar, der die Handlungsausführung lenkt (Scholz und Binder 2004, S. 3). Scholz (2011) konzeptualisiert humane Systeme daher als planvoll handelnd, die ihre Strategien bewusst nach deren Nützlichkeit für das Ziel auswählen (Scholz 2011, S. 443; Scholz und Binder 2004, S. 3). Weiterhin ist das Handeln humaner Systeme auch durch Lernprozesse geprägt, die aus der Evaluation von (i) geplantem Handeln, (ii) geschehenen Handeln oder der (iii) Umweltreaktion resultieren können (Scholz und Binder 2003, S. 6)

- P6 Umweltwahrnehmung \& -bewusstsein: Humane Systeme nehmen ihre Umwelt wahr und diese Wahrnehmung hat einen entscheidenden Einfluss auf ihre Ziele, Strategiewahl, Handlung und ihre Lernprozesse. Scholz und Binder (2003) schlagen drei Archetypen des Umweltbewusstseins vor, die aus der Umweltwahrnehmung resultieren: umwelt-ignorant (die Effekte des eigenen Handelns auf die Umwelt werden nicht wahrgenommen, kein Einfluss auf die individuellen Ziele und Strategien), umwelt-bewusst (Rückkopplungen erster Ordnung zwischen der Umwelt und dem menschlichen Verhalten werden wahrgenommen, Ziel und Strategiebildung werden von der Umweltwahrnehmung beeinflusst) und umwelt-geleitet (auch Rückkopplungen zweiter Ordnung werden wahrgenommen, Ziele und Strategien werden maßgeblich beeinfluss, was in einem altruistischen Handeln münden kann, das heißt eigene Ziele und Präferenzen werden zur Vermeidung negativer Umwelteffekte zurückgestellt) (Scholz 2011, S. 444; Scholz und Binder 2003, S. 6)

- P7: Umwelt zuerst: Scholz (2011) schlägt vor, dass jede Analyse eines Mensch-Umwelt Systems mit einer präzisen Systemanalyse des Umweltsystems beginnen sollte, in der Struktur, Mechanismen und Rückkopplungen genau verstanden werden, um darin die weiterführende Analyse des Handelns im humanen System einbetten zu kommen. Dieses Postulat wird von Scholz selbst auch als normativ aufgeladenes bezeichnet, das klar den Ursprung des HES-Framework in den Nachhaltigkeit-, bzw. Umweltwissenschaften aufzeigt und nicht nur zum Verständnis, sondern auch zur besseren Lösung von MenschUmwelt Problemen beitragen will (Scholz 2011, S. 448). 
Tab. 1 Ebenen des sozialen, ökologischen und technologischen Subsystems. (Eigene Darstellung)

\begin{tabular}{lll}
\hline Soziales Subsystem & Ökologisches Subsystem & Technologisches Subsystem \\
\hline Individuum & Individuum/Gemeinschaft & $\begin{array}{l}\text { Technologische Komponenten } \\
\text { des Artefakts }\end{array}$ \\
$\begin{array}{l}\text { Gruppe } \\
\text { Organisation }\end{array}$ & $\begin{array}{l}\text { Technologisches Artefakt } \\
\text { Technologie }\end{array}$ \\
$\begin{array}{l}\text { Institution } \\
\text { Nationale Gesellschaft }\end{array}$ & Ökosystem & Technologiegruppe \\
Supranationale-Gesellschaft & Landschaft/Ökoregion & \\
\hline
\end{tabular}

\section{Adaptation des HES Frameworks für sozio- technische Systeme}

\subsection{Erweitertes Systemverständnis}

Wie bereits in der Einleitung erwähnt, basiert das HES Framework auf der Annahme sozio-ökologischer Systeme, geht in der Konzeption des Systems jedoch wie auch die Studien zu sozio-technischen Systemen von ,complex systems“ aus, sodass eine Übertragung naheliegt, zumal einerseits sozio-technische Systeme ökologische Komponenten als Ressourcen mit in das technologische Subsystem integrieren (Geels, 2004) und andererseits Scholz und Binder (2004) ein eher breit angelegtes Umweltverständnis zugrunde legen: „Human-Environmental Systems include all environmental and technical systems that are relevant for and affected by humans“ (Scholz und Binder 2004, S. 1). Scholz und Binder (2004) inkludieren technologische Komponenten somit in ihrer Definition von Mensch-Umweltsystemen, dennoch sind technologische Aspekte nicht weiter explizit konzeptualisiert, da das Forschungsinteresse zur Entwicklung des HES Frameworks ursprünglich vorwiegend in der Analyse des Einflusses von Umweltwahrnehmungen auf das Akteurshandeln lag. Für die Transition sozio-technischer Systeme spielen diese ökologischen Aspekte zwar ebenfalls eine wichtige Rolle, dennoch sind Wahrnehmungen der technologischen und sozialen Umwelt mindestens ebenso wichtig. Beispielsweise beeinflusste der Reaktorunfall in Fukushima als technologischer Faktor die Energiepolitiken und den Fortschritt der Energiewende in Deutschland und Zentraleuropa entscheidend, wie dagegen der Preiszerfall von fossilen Brennstoffen und Elektrizität als sozialer Faktor die Energiewende in jüngster Zeit vor Schwierigkeiten stellte. Wir schlagen daher vor, die Termini Umwelt und Umweltwahrnehmung für die Anwendung des HESFrameworks auf sozio-technische Systeme in Umfeld und Umfeldwahrnehmung zu wandeln, um damit das erweiterte Systemverständnis zu verdeutlichen und Missverständnissen vorzubeugen (vgl. Abb. 2).

Für die Anwendung des HES Frameworks auf soziotechnische Systeme, konzeptualisieren wir somit ökologische Elemente als in das technologische Subsystem eingebettete Ressourcen und integralen Bestandteil des sozio-technischen Systems (vgl. sozio-technisches Systemverständnis (Geels 2004)). Dieses sozio-technische und ökologische System wird dabei, wie von Scholz (2011) und Geels (2004) vorgeschlagen, ebenfalls als komplexes, auf unterschiedlichen Skalenebenen interdependentes und komplementäre Subsysteme umfassendes System konzeptualisiert (vgl. Postulat 1).

\subsection{Mehr-Ebenen Konzept}

Vergleichbar zu den von Scholz (2011) vorgeschlagenen Ebenen des sozialen und ökologischen Subsystems kann auch das technologische Subsystem in mehrere Ebenen untergliedert werden (Postulat 2). Für Energiesysteme schlagen wir folgende Ebenen vor: technologische Komponenten, technologisches Artefakt, Technologie und Technologiegruppe. Die unterste Ebene der technologischen Komponenten umfasst alle Komponenten, die zur Funktion des technologischen Artefakts nötig sind. Im Falle einer Wasserkraftanlage z. B. Turbinen, Leitungen, Dämme etc. Die Ebene des technologischen Artefakts umfasst für den Fall des Energiesystems die einzelnen Produktionsanlagen (wie z. B. ein Windrad), die Ebene der Technologie fasst alle Anlagen einer Technologie (z. B. PV-Dachflächen und Freiflächenanlagen) zusammen und die Technologiegruppe fasst mehrere Technologien z. B. zur Strom- oder Wärmeproduktion, bzw. netzgebundene oder nicht Netzgebundene Technologien zusammen. Listet man die Ebenen aller drei Subsysteme gemeinsam auf (vgl. Tab. 1) wird deutlich, dass die Aggregationsschritte für die einzelnen Subsysteme - aufgrund ihrer Eigengesetzlichkeit (Postulat 1) - jedoch nicht gleich groß sind und somit nicht zu jedem Level jedes Subsystems ein Äquivalent in den anderen Subsystemen definiert werden kann.

Dennoch ist es hilfreich diese Untergliederung für jedes einzelne Subsystem vorzunehmen, da Umfeldwahrnehmungen (Postulat 6), Umfeldreaktionen (Postulat 4) und Interferenzen (Postulat 3) innerhalb aber auch zwischen den Subsystemen somit präzisiert, lokalisiert und analysiert werden können. So kann z. B. die deutschlandweit virulente Windkraftproblematik untergliedert werden in Aspekte, die das Technologische Artefakt (das einzelne Windrad) oder die Technologie (Windkraft) betreffen aber auch dessen Aus- 
wirkung auf bestimmte ökologische Individuen (z. B. Rotmilan) und die Reaktionen im sozialen Umfeld auf Ebene der Gruppe (Windkraftgegner) sowie der Institution (bayerische Abstandregelung für Windräder).

\subsection{Soziale Interferenzmechanismen}

Mit diesem erweiterten Systemverständnis wird jedoch nicht nur das technologische Subsystem in die Betrachtung integriert, sondern es erfolgt auch eine stärkere Berücksichtigung der Mechanismen auf und zwischen den unterschiedlichen Ebenen im sozialen System. Im von Scholz (2011) vorgeschlagenen HES Framework, ist das gesellschaftliche Subsystem zwar nach seinen Regelmechanismen in unterschiedlichen Ebenen gegliedert (,Hierarchieebenen“ (Postulat 2)) und mit dem Konzept der Interferenz auch die Interaktion zwischen den gesellschaftlichen Ebenen angelegt (Postulat 3), doch hat sie in den bisherigen Anwendungen des HES keine größere Berücksichtigung gefunden (Binder et al. 2013; Scholz und Binder 2003, 2004, 2011). Wir schlagen daher mit unserem Umfeld-Begriff auch eine dezidiertere Berücksichtigung und genauere Analyse der Interferenzmechanismen zwischen unterschiedlichen gesellschaftlichen Ebenen vor (z. B. Institutionalisierung, Strukturation etc.). Für die Energiewende meint das z. B. die deutschlandweite Gründung von Vereinen für die Förderung erneuerbarer Energien oder die Gründung von Bürgerenergiegenossenschaften, aber auch die Entwicklung nationaler Politiken, wie das Erneuerbare-Energien-Gesetz oder der Entscheid zum Atomausstieg. Diese explizite Berücksichtigung der Interferenzmechanismen zwischen den sozialen Eben ermöglicht ein besseres Verständnis der gesamtgesellschaftlichen Veränderungsprozesse und schafft die Basis für eine bessere Anschlussmöglichkeit des HES Frameworks an die Arbeiten der Transitionsforschung, worauf wir in der Diskussion noch vertiefter eingehen werden.

Wir schlagen somit eine gleichwertige Betrachtung gesellschaftlicher, technologischer und ökologischer Aspekte sowie eine Berücksichtigung der Interferenzen in allen Subsystemen vor. Dies ermöglicht auch eine direkte Vereinbarkeit des Frameworks mit dem Leitbild Nachhaltiger Entwicklung. Dementsprechend schlagen wir auch vor Postulat 7 (Umwelt zuerst) hin zu ,detaillierte Umfeldkenntnis“ abzuschwächen, denn eine profunde Kenntnis der Systemmechanismen ist zur Analyse und dem Verständnis der Rolle des Akteurshandelns entscheidend, nur sehen wir von einer stärkeren Gewichtung des ökologischen Subsystems $a b$ und plädieren für eine gleichwertige Analyse des sozialen, technologischen und ökologischen Umfeldes.

Abgesehen von diesen Veränderungen bezüglich des Systemverständnis kann die Konzeptualisierung des Akteursverhaltens und seiner Interaktion mit dem systemi- schen Umfeld für eine Anwendung auf sozio-technische Systeme beibehalten werden. Diese hat sich für die Analyse des individuellen Akteurshandelns in der regionalen Energiewende im bayerischen Allgäu als sehr produktiv erwiesen und verändert sich nicht bei erweitertem Systemverständnis.

\section{Anwendung des HES Frameworks auf sozio- technische Systeme - Fallbeispiel Change Agents in der Allgäuer Energiewende}

Im Folgenden wenden wir das HES Framework unter der Berücksichtigung der vorgeschlagenen Erweiterungen auf die Energiewende im bayerischen Allgäu an. Dazu stellen wir zunächst die Entwicklungen des Energiesystems im bayerischen Allgäu vor sowie das untersuchte Netzwerk der sog. Change Agents, die die Energiewende in der Region entscheidend vorangetrieben haben. Anschließend erläutern wir unser methodisches Vorgehen und präsentieren exemplarische Ergebnisse unserer Fallstudie für die Anwendung des HES Frameworks auf das Energiesystem.

\subsection{Die Untersuchungsregion bayerisches Allgäu}

\subsubsection{Die Region}

Das bayerische Allgäu gilt als eine der Pionierregionen Deutschlands hinsichtlich des Fortschritts der Energiewende: Der Anteil erneuerbarer Energien am Gesamtstromverbrauch liegt im Allgäu bei ca. $49 \%$ (Bayern $35 \%$, Deutschland $28 \%$ (BSTMWI 2016)) und erste Initiativen und Vereinsgründungen finden sich in der Region schon in den 1990er-Jahren - weit vor dem Erneuerbare-Energien-Gesetz (EEG) 2001. Vor allem aber hinsichtlich des aus der lokalen Gesellschaft heraus getragenen Energiewende-Engagements und des Beitrags einzelner Individuen zum Transformationsprozess ist das Allgäu bekannt. Zahlreiche mittelständische Unternehmen sowie Bürgergenossenschaften sind an der Produktion und Weiterentwicklung erneuerbarer Energien beteiligt, viele Vereine vertreten die Interessen der Akteure und einzelne Individuen stehen im Zentrum der Transition.

Die Region umfasst die vier Landkreise Ost-, Ober- und Unterallgäu sowie Lindau und die kreisfreien Städte Memmingen, Kaufbeuren und Kempten und liegt am südwestlichen Alpenrand Bayerns (vgl. Abb. 3).

Abb. 4 zeigt für die Untersuchungsregion den generellen Anstieg von Produktionsanlagen für erneuerbare Energien vor allem aber einen enormen Zubau der Anlagen zur Stromproduktion aus Sonnenenergie. Von 2000 bis 2013 haben diese von einigen wenigen auf über 35.000 Anlagen mit einer gesamt installierten Leistung von rund $800 \mathrm{GWh}$ 


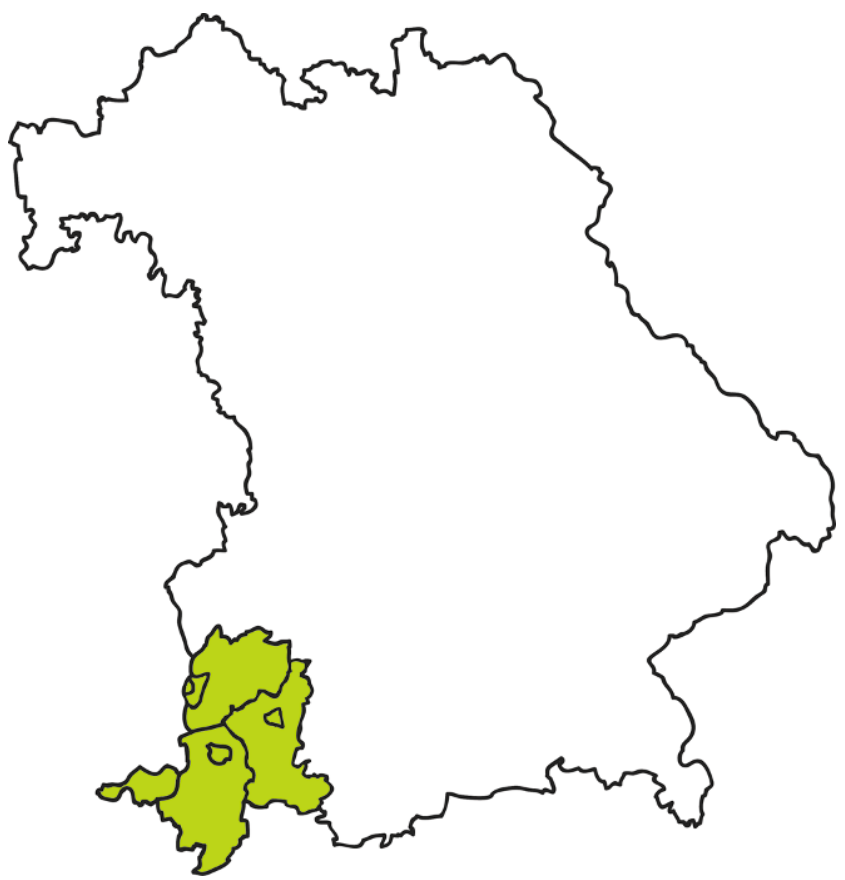

Abb. 3 Die Untersuchungsregion „bayerisches Allgäu“. (Eigene Darstellung)

im bayerischen Allgäu zugenommen. Auch bei den Anlagen zur Stromproduktion aus Wasserkraft und der Biomassenutzung ist ein deutlicher Zuwachs erfolgt, wobei die Ausgangslage bei der Wasserkraft im Jahr 2000 mit knapp 200 Anlagen deutlich höher war als bei der Biomasse (knapp 50 Anlagen). Bis 2013 wurde der Anlagenpark zur Wasserkraft- und Biomassenutzung je auf fast 350 Anlagen ausgebaut. Deutlicher zeigt sich der Zuwachs der Biomassenutzung noch bei der Installierten Leistung (vgl. Abb. 4b): von ca. $30 \mathrm{GWh}$ wurde dieser auf etwa $380 \mathrm{GWh}$ erhöht. Bei der traditionell in der Region stark genutzten Wasserkraft fiel der Zuwachs mit etwa $200 \mathrm{GWh}$ zwischen 2000 und 2013 geringer aus. Die Windkraft erfuhr einen verhalteneren Ausbau auf etwa $90 \mathrm{GWh}$. Klärgase bleiben nur sehr gering und Geothermie ungenutzt.
Bezüglich der Umfeldreaktion im sozialen Subsystem wurden in Abb. 5 exemplarische „Meilensteine“ des Institutionalisierungsprozesses zu erneuerbaren Energien in der Region zusammengestellt. Es zeigt sich deutlich, dass sich die regionale Energiewende nicht nur in Vereins- und Firmengründungen manifestierte, sondern der Fortschritt auch in Form der Schaffung neuer Stellen (Energiemanager), der Auszeichnung von Gemeinden (z. B. Energiegemeinde) und der Schaffung regionaler Klimaschutz- und Energiewendeleitbilder sichtbar wurde.

Es wird deutlich, dass in der Region in den 1990er und 2000er-Jahren eine enorme Beschleunigung der Energiewende im sozialen und technologischen Subsystem erfolgt ist und somit das Umfeld auf die Handlungen der Change Agents enorm positiv reagiert hat.

\subsubsection{Die Akteure}

Die in unserer Studie befragten zentralen Change Agents der Region sind in den Bereichen Land- und Forstwirtschaft, Handwerk (Elektro- und Lüftungstechnik), Energieberatung, Energie- und Abfallwirtschaft sowie Regionalentwicklung und Bankenwesen tätig (vgl. Abb. 6). Die meisten verfügen über eine technische Ausbildung, bzw. ein Ingenieursstudium und sind zusätzlich ehrenamtlich engagiert. Sie stammen entweder gebürtig aus der Region Allgäu oder leben schon seit mehr als 10 Jahren dort. Die meisten leben und arbeiten in und um Kempten, dem Oberzentrum der Region, wo auch viele Organisationen, Unternehmen sowie Politik und Verwaltung angesiedelt sind. Alle Akteure sind männlich und im Alter zwischen 40 und 65. Die meisten unter ihnen können als sog. Entscheidungsträger bezeichnet werden, da sie entweder Unternehmensinhaber, Geschäftsführer, Bürgermeister oder Landräte, Vereinsvorstände oder Leiter einer Organisation sind.

Großteils kennen sich die befragten Akteure persönlich und seit langen Jahren. Dadurch, dass die meisten auch in mehreren (oft ehrenamtlichen) Positionen tätig sind, sind sie untereinander mehrfach und stark vernetzt. Sie ver-
Abb. 4 Ausbau der erneuerbaren Energien im Allgäu. (Quelle: Deutsche Gesellschaft für Sonnenenergie e. V. [o. J.]; Datenbestand vom: 21.02.2014, eigene Darstellung)

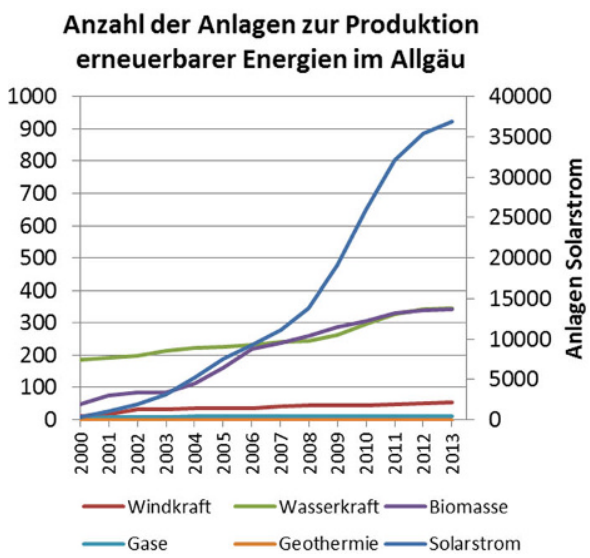



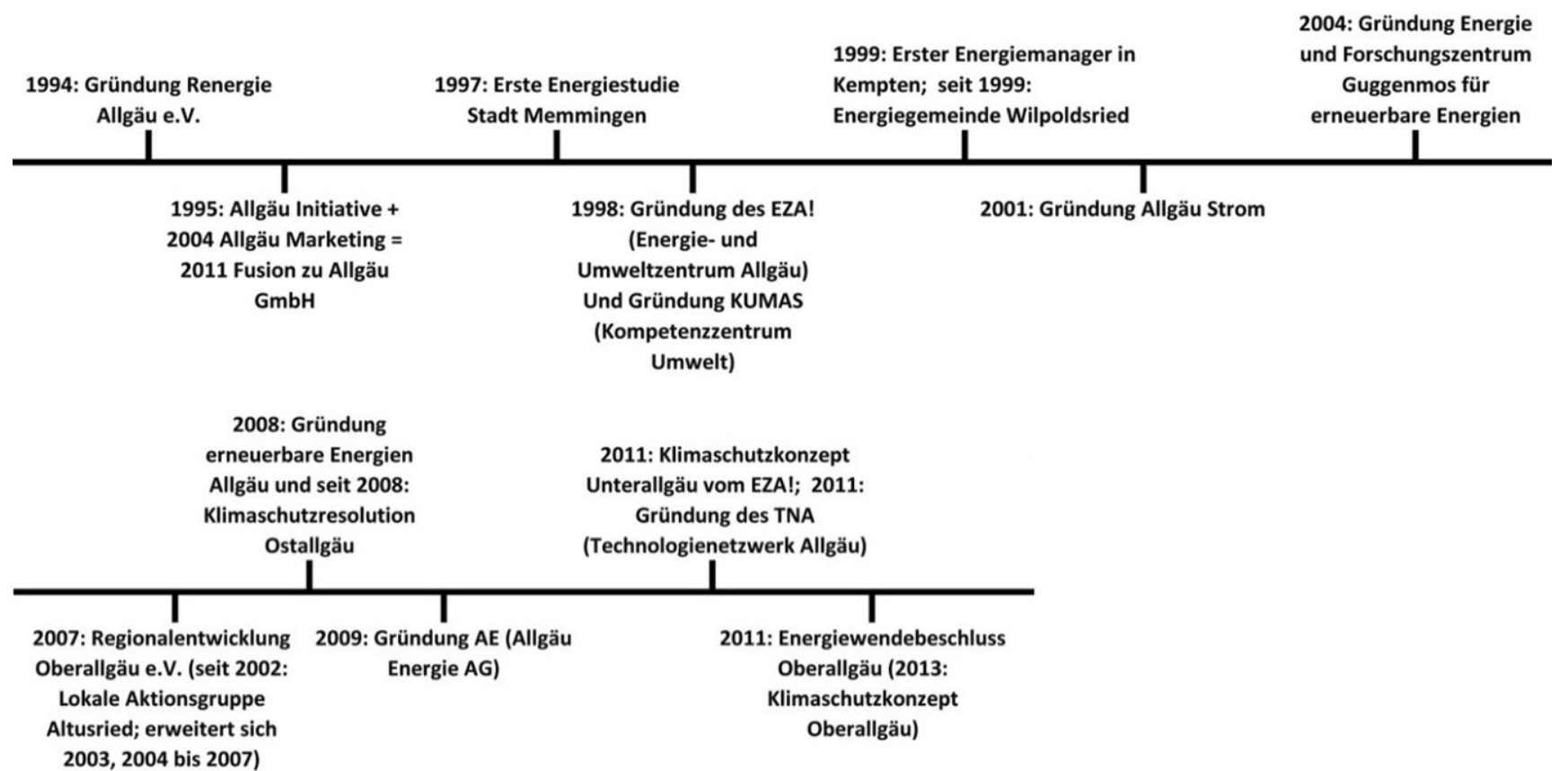

Abb. 5 Institutionalisierungsprozess im Allgäu. (Eigene Darstellung)
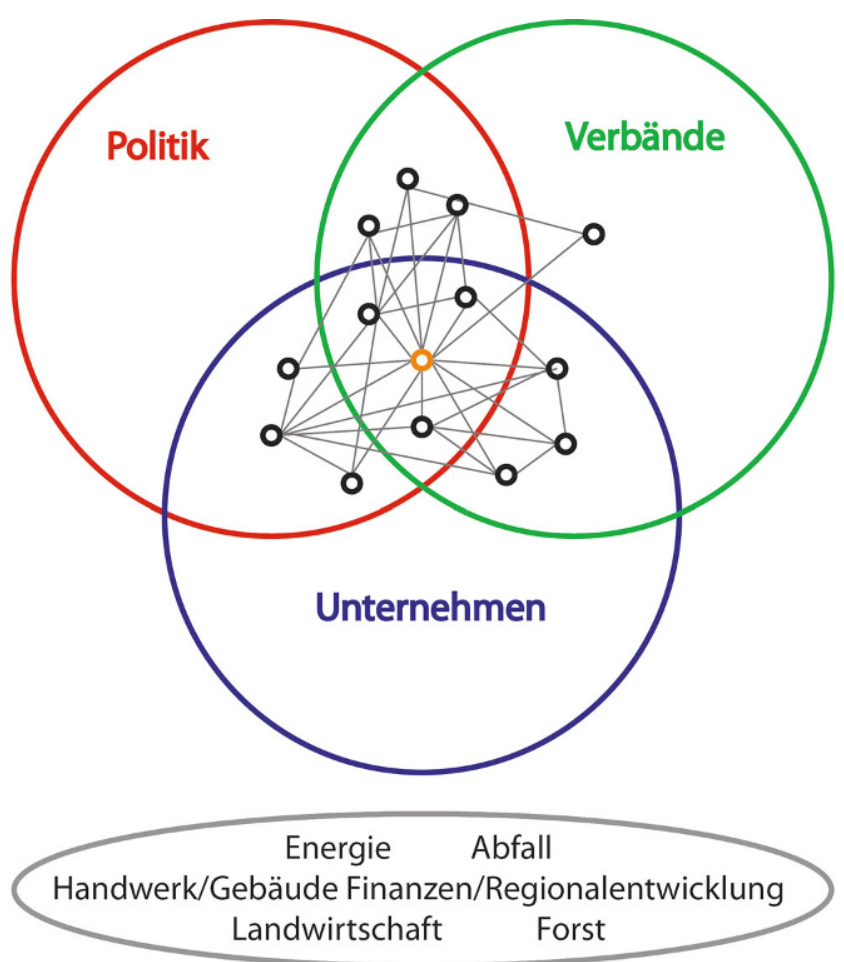

Abb. 6 Netzwerk der zentralen Akteure der Allgäuer Energiewende. (Eigene Darstellung)

binden durch diese Ämterhäufung unterschiedliche gesellschaftliche Bereiche in einer Person: viele sind als Politiker auch Unternehmer und Vereinsvorstand und andersherum (vgl. Überlappungsbereiche in Abb. 6). Die Akteure treffen daher je nach Kontext (ob in der Politik oder im Verein) in unterschiedlichen Funktionen und Konstellationen immer wieder aufeinander. Im Zentrum dieses Akteursnetzwerks steht das Umwelt- und Energiezentrum Allgäu (eza!) ${ }^{1}$, da hier alle Akteure in unterschiedlichster Weise aktiv, beteiligt oder affiliiert sind (vgl. oranger Punkt im Zentrum in Abb. 6).

Alle Befragten haben im Laufe der letzten 20 Jahre zu technischen und/oder sozialen Innovationen in der Region beigetragen: es wurden neue Antriebstechniken, Energiegewinnungsprozesse oder Verteilnetzstrukturen entwickelt, ebenso wie neue finanzielle Beteiligungsmodelle, Veranstaltungs- und Vernetzungsformate oder neue Institutionen gegründet.

\subsection{Methodisches Vorgehen}

Als räumliche Ebene unserer Fallstudie wurde „die Region“ (sieben Landkreise) gewählt, da somit nicht nur die lokale, unmittelbare Wirkungsebene der Akteure (Kommune), sondern auch ihre Einbettung in die übergeordnete Skalenebene integriert und mögliche Interferenzen berücksichtigt werden konnten (Regierungsbezirk, Bundesland). In 2014/2015 wurden insgesamt 14 explorative Experteninterviews mit zentralen Change Agents der regionalen Energiewende im bayerischen Allgäu geführt. Als Change Agents wurden dabei Akteure definiert, die in der Region maßgeb-

\footnotetext{
1 eza! ist eine gemeinnützige GmbH zur Förderung erneuerbarer Energien und effizienter Energienutzung und wird getragen von Kommunen, Wirtschaft und Initiativen des Allgäus (http://www.eza-allgaeu. de/ueber-eza/).
} 
lich zur Pionierphase der Energiewende beigetragen hatten. Darunter fielen Akteure, die entweder technische und soziale Innovationen geschaffen hatten (z. B. Ingenieure, Unternehmensgründer, Vereinsgründer) oder bei deren Implementation unterstützend tätig waren (z. B. Politiker, Unternehmer). Die Interviews wurden nach dem sog. Schneeballverfahren (snowball sampling) geführt: Ausgangspunkt der Befragung (point of entry) waren dabei ein lokal sehr gut vernetzter Banker, zu dem ein persönlicher Kontakt bestand sowie ein Mitarbeiter der regionalen Energiewendeorganisation, die aus der Sekundärdatenanalyse als wichtigste Organisation in der Region ermittelt wurde. Beide wurden nach weiteren zentralen Change Agents der Pionierphase der regionalen Energiewende gefragt, die wiederum in ihren Interviews nach weiteren Namen gefragt wurden, bis sich der Kreis der genannten Akteure geschlossen hatte. In den leitfadengestützten Experteninterviews wurden (i) die Motive für das Engagement und die Wahrnehmung der eigenen Rolle im Energiewendeprozess, (ii) der wahrgenommene Verlauf der Energiewende und (iii) die als förderlich oder hinderlich wahrgenommene Einflussfaktoren erfragt. Zur Auswertung der transkribierten Interviews wurden die Aussagen der Befragten mit MAXQDA softwarebasiert kodiert (www.MAXQDA.de) und inhaltlich kategorisiert. Es wurde dabei ein qualitativ-strukturierter Analysezugang gewählt, bei dem die Transkripte nach den Kategorien des angepassten HES Frameworks - (i) Ziele/Motive der Akteure, (ii) Strategien und (iii) positive und hinderliche Umfeldwahrnehmung auf unterschiedlichen Ebenen - sortiert wurden (strukturierende qualitative Inhaltsanalyse (Mayring 2010)). Weiterhin wurden öffentlich verfügbare Sekundärdaten zum technischen Ausbau und dem gesellschaftlichen Institutionalisierungsprozess der erneuerbaren Energien in der Region hinzugezogen, um die soziale und technologische Umfeldreaktionen zu untersuchen. Für das technologische Subsystem wurden öffentlich verfügbare Daten zum Anteil der erneuerbaren Energien auf Landkreisebene analysiert (vgl. energymaps.info) sowie die Ergebnisse einer regionalen Energieflussanalyse hinzugezogen (vgl. Mühlemeier et al. in press). Für die Analyse des Institutionalisierungsprozesses wurden Vereins- und Organisationsgründungen in der Region auf Basis einer online-Recherche nachgezeichnet. Die Ergebnisse dieser Studie werden im Folgenden exemplarisch dargestellt.

\subsection{Exemplarische Anwendung des HES Frameworks auf die regionale Energiewende im bayerischen Allgäu}

Im Folgenden präsentieren wir exemplarisch einige der Ergebnisse aus der oben genannten Studie zur regionalen Energiewende im bayerischen Allgäu, um die Anwendung des HES Frameworks auf sozio-technische Systeme zu illustrieren.

\subsubsection{Umfeldwahrnehmungen und Ziele: die Handlungsmotive der Change Agents}

Die für das Engagement in den Anfängen der regionalen Energiewende im Allgäu in den 1990er und frühen 2000erJahren genannten Handlungsmotive der befragten Change Agents können in Abhängigkeit von den genannten Umfeldwahrnehmungen in drei Typen untergliedert werden:

- Die meisten Akteure nannten Motive im Bereich ,grundlegende Überzeugung für die Wichtigkeit des Ausbaus der erneuerbaren Energien“. Diese Zielkategorie wurde einerseits im Zusammenhang mit der Wahrnehmung globaler Umweltprobleme, wie z.B. der Klimaerwärmung oder der Endlichkeit fossiler Ressourcen genannt, andererseits aber auch mit der Wahrnehmung technologischer Probleme, wie der Möglichkeit eines Reaktorunfalls und der Problematik des Ersetzens des Anteils der Kernkraft an der Stromproduktion durch erneuerbare Energien und schließlich mit der eher gesellschaftlichen Sorge um die Lebensbedingungen zukünftiger Generationen (,wenn einen die Zukunft der Menschheit interessiert, dann beschäftigt man sich automatisch mit energiepolitischen Fragen“(A1))

- Die zweite große Zielkategorie konnte im Bereich ,technische Optimierung der Energieversorgung “aus regionalen Quellen und „Engagement in der Region“ gruppiert werden. Diese Zielkategorie wurde im Zusammenhang mit der Wahrnehmung lokal vorhandener Technologien zur Produktion von Energie aus regional verfügbaren erneuerbaren Energien erwähnt, sowie der Sorge um die regionale Entwicklung des Allgäus, die regionale Wertschöpfung und Möglichkeit in dieser Region leben und arbeiten zu können.

- Schließlich konnten zwei weitere Zielkategorie im Bereich „Entwicklung von Geschäftsmodellen“ in der Energiewende ermittelt werden, die damit vorwiegend ökonomische Motive umfasste sowie Motive im Bereich ,etwas praktisch gestalten und umsetzen“, die vor allem die eigene Wirksamkeit (vor Ort) betonte. Im Zusammenhang mit diesen Motiven wurden keine direkten Umfeldwahrnehmungen genannt.

Diese drei Bereiche der Zielkategorien spiegeln die von Scholz (2011) vorgeschlagene Typologie zum Grad der Umfeldwahrnehmung wider, wobei die letzte Gruppe auf eine geringe bis nicht vorhandene Wahrnehmung von Umfeldeinflüssen hindeutet, die zweite Gruppe deutet auf Umfeldwahrnehmungen erster Ordnung hin (direkte Effekte) und die erste Gruppe zeigt Wahrnehmungen von vorhandenen und potenziellen Rückkopplungen zweiter 
Ordnung (zeitlich weiter entfernt). Die Akteure nannten Ziele aus mehreren Bereichen gleichzeitig, sodass die Zielbereiche pro Akteur überlappen und keine Typenbildung der untersuchten Akteure nach den Zielbereichen und Umfeldwahrnehmungen vorgenommen werden kann.

\subsubsection{Strategien und Handlungen der Change Agents}

Für die Strategien und Handlungsformen der Change Agents in der Pionierphase der Energiewende konnten vier Strategie- und Handlungsbereiche ermittelt werden. Aufgrund der in den Interviewleitfäden jedoch noch nicht vorgesehenen expliziten Unterscheidung zwischen Strategiewahl und erfolgter Handlung, muss dieser Bereich hier zusammengefasst betrachtet werden.

- Gut zwei Drittel der Befragten nannte Handlungsformen und Strategien im Bereich Wissensaufbau und gezielte Wissensvermittlung durch kontinuierliche Erweiterung des eigenen Wissens, die Schulung der Mitarbeiter (zum Teil durch gezielte außerregionale Vernetzung) oder die Information der Bevölkerung (z. B. durch Aufbau von Energieberatungsagenturen, regionalen Messen und Informationsveranstaltungen). Weiterhin fielen hierunter auch Handlungsformen wie Vereins- und Netzwerkgründungen, der gezielte Austausch und das sich Vernetzen mit regionsexternen Experten und politischen Instanzen (z. B. in EU-Fachkreisen).

- Etwa die Hälfte der Befragten nannte Handlungsformen und Strategien, die sich im Bereich Bildung strategischer Allianzen zusammenfassen ließen. Genannte Strategien waren hierbei z. B. die Pflege des persönlichen Netzwerks, gezieltes Einbinden von regionalen Schlüsselpersonen aus Politik und Wirtschaft sowie die Beteiligung der regionalen Bevölkerung zur Schaffung grundlegender Akzeptanz und eines tragfähigen regionalen Netzwerks, welches das Engagement zur Energiewende unterstützte.

- Ein Drittel nannte Strategien und Handlungen im Bereich Mobilisierung regionalen Kapitals, z. B. durch finanzielle Beteiligung der regionalen Bevölkerung, forcierte Kooperation mit regionalen Energieversorgern und Unternehmen (z. B. regional beschränkte Ausschreibungen) oder der Gründung von Bürgerenergiegenossenschaften mit kleinen Anteilen; auch um Unabhängigkeit von großen Geldgebern zu schaffen. Darunter fiel z. B. auch die Entwicklung erster Finanzprodukte im Bereich PVFinanzierung, die später auch außerhalb des Allgäus übernommen wurden.

- Abschließend nannten alle befragten Handlungen, die sich im Bereich Innovationen schaffen zusammenfassen ließen und natürlich mit der Auswahl der Change Agents zusammenhing. Sie alle hatten entweder zur Entwick- lung technologischer Innovationen (z. B. eines Hauses, das sich nach der Sonneneinstrahlung ausrichtet oder einer optimierten Müllverbrennungstechnologie), dem Errichten neuer Produktionsanlagen (Windkraft, Biogasanlagen) oder der Gründung von eigenen Firmen im Bereich technologischer Lösungen zu erneuerbaren Energien oder deren Vertriebsmöglichkeiten (z. B. regionaler Holzvermarktungsverbund) beigetragen.

Zusammenfassend lässt sich festhalten, dass die Handlungsformen sowohl im technologischen als auch im sozialen Subsystem erfolgten und dabei auf unterschiedlichen Hierarchieebenen: von der Ausbildung oder Beteiligung von Individuen, hin zur Gründung von Gruppen und Organisationen, bzw. von dem Bau einer Produktionsanlage hin zur Entwicklung einer neuen Technologie. Durch die eingangs bereits erwähnte Mehrfacheinbettung und das vielseitige Engagement der Change Agents, konnten für dieses Akteursnetzwerk keine Typen gebildet werden, wonach bestimmte Akteure nur in einem bestimmten Handlungsbereich tätig wären. Vielmehr scheinen die Change Agents auf unterschiedlichen Ebenen und in unterschiedlichen Bereichen des Energiesystems zu wirken.

\subsubsection{Umfeldreaktionen, Interferenzen und Evaluationen der Change Agents}

Neben der Kenntnis der allgemeinen Umfeldreaktionen bzw. der generellen Systementwicklungen (Postulat 7), die im vorherigen Abschnitt zum Fallstudienbeschrieb (Abschn. 4.1.1) bereits erläutert wurden, legt das HES Framework den Analysefokus aber vor allem auch auf die von den Change Agents wahrgenommenen Umfeldreaktionen, die im Zusammenhang mit ihrem Handeln stehen.

Im Kontext dieser exemplarischen Anwendung konnte zwar noch keine detaillierte Erhebung der wahrgenommenen Umfeldreaktionen auf die einzelnen Handlungen der Change Agents vorgenommen werden, dennoch können im Folgenden einige exemplarische Beispiele für positiv und negativ wahrgenommene Umfeldreaktion genannt werden.

- Als positive Umfeldreaktionen wurden auf Ebene des Individuums bzw. der Gruppe der starke Zusammenhalt unter den Change Agents, die positive Resonanz aus diesem Akteursnetzwerk und dem weiteren persönlichen Umfeld der Change Agents genannt, die sie in ihrem Handeln bestärkten. Auf der Ebene der Organisation konnten viele Kommunen von der Investition in erneuerbare Energien (energetische Sanierung ihrer Liegenschaften) und der Genehmigung von Anlagen (Fernwärmenetze, Windkraft) überzeugt werden. Die von den Change Agents angestrebte regionale Vernetzung von Unternehmen und Organisationen im Bereich erneuerba- 
re Energien, z. B. zu Produktionstechnologien aber auch verwandte Sektoren, wie der Gebäudesanierung, wurde von den regional ansässigen Unternehmen angenommen und entsprechende Netzwerke konnten entstehen. Dies geschah auch mit der Unterstützung und positiven Resonanz der regionalen Wirtschaftsverbände. Im Kontext dieser Netzwerke konnten in den Anfängen erste Pilotanlagen (z. B. zu Müllverbrennung) in Kooperation mit dem regionalen Energieanbieter realisiert werden, und später sowohl einige Firmen zur Energieproduktion (v.a. aus Biomasse und PV) durch die Change Agents und regionale Partnerfirmen gegründet werden, als auch größere Forschungsprojekte in der Region mit externen Partnern realisiert werden (z.B. zur Erforschung intelligenter Netze mit hohen Anteilen an erneuerbaren Energien). Auf der Ebene der Institutionalisierung wurden von den Change Agents hervorgehoben, dass etliche Kommunen die Klimaschutz- und Energiewendepolitiken übernommen hätten, die von Ihnen initiiert wurden und z. B. eben regionale Klimaschutzmanagerund Energieberatungsstellen geschaffen wurden. Ein anderer Change Agent berichtete, wie die von ihm entwickelten Finanzierungsmodelle für PV Anlagen nicht nur regional von den Kommunen, sondern auch überregional übernommen wurden. Aktive Kommunen wurden darüber hinaus auch mit etlichen Preisen ausgezeichnet (z. B. Klimaschutzpreise oder dem european energy award). Auf der Ebene der regionalen Gesellschaft wurde hervorgehoben, dass viele Allgäuer sich an Energieproduktionsgenossenschaften finanziell beteiligt hätten und somit nicht nur die Finanzierung von den Change Agents initiierten Projekte ermöglicht haben, sondern zudem auch eine breite Akzeptanz für die Energiewende in der Region als positive Umfeldreaktion evaluiert wurde (vgl. auch Mühlemeier und Knöpfle 2016).

- Demgegenüber wurden von den Change Agents jedoch auch einige negative Umfeldreaktionen wahrgenommen, die im direkten Zusammenhang mit ihrem Handeln standen. Auf der Ebene der regionalen Gesellschaft war oftmals das erhoffte Publikum für die initiierten Informationsveranstaltungen und Messen ausgeblieben, was eine gewisse Frustration bei den Change Agents hervorrief, ebenso wie die oftmals langwierigen Diskussionen in Gemeinderatssitzungen zur Umsetzung der Klima- und Energiepolitiken sowie der Genehmigungen von Produktionsanlagen. Auf Ebene der Organisationen wurden besonders sachlich falsche Medienberichte über die erneuerbaren Energien im Allgemeinen oder aber „hämische“ Berichte über gescheiterte Pilotprojekte als negative Umfeldreaktion wahrgenommen, die das eigene Engagement besonders erschwerten. Manche Change Agents reflektierten auch über die Problematik, dass ihr Unternehmen oder ihre Organisation stark gewachsen sei und nun mit Personalwechseln und Neueinstellung das Problem der Wissensweitergabe und des Commitments zum Energiewendeengagement als negatives Feedback ihre Arbeit erschwere. Weiterhin wurde von einem Change Agent auch der massive Widerstand etablierter Großunternehmen in der Region gegen eine von ihm mit initiierte lokale Ressourcenvertriebsstruktur genannt, der ihm große Steine in den Weg gelegt hatte. Zuletzt wurde das entstehen regionaler Gegnergruppierungen (,Wutbürger“) v. a. gegen Windkraftanlagen genannt, die als Reaktion auf den von den Change Agents vorangetriebenen Ausbau der Windkraft in der Region gegründet wurden und ein Voranschreiten des Ausbaus verlangsamten oder blockierten.

Zusätzlich zu den aufgeführten Umfeldreaktionen, nannten die Change Agents auch weitere Umfeldeinflüsse, die zwar nicht im direkten Zusammenhang mit ihrem Handeln standen, dennoch von den Change Agents als entscheidende Einflussfaktoren evaluiert wurden. Es handelt sich dabei um Entwicklungen, die im Kontext der Energiewende auf übergeordneten Ebenen des sozialen Systems lokalisiert wurden und einen Einfluss auf die untergeordnete Ebene des Akteurshandelns hatten. Diese Interaktion zwischen den Ebenen des sozialen Subsystems können daher als Interferenzen verstanden werden können (Postulat 3).

- Als positive Interferenz von Ebene der Gesellschaft, bzw. der Institutionen nannten die Akteure die bundesweit etablierten Fördermaßnahmen für die Energiewende, die zwar nicht als direkte Umfeldreaktion auf ihr Handeln gesehen werden können, dennoch als enorm wichtige und fördernde Faktoren wahrgenommen wurden, die ihr Engagement in der Region, vor allem aber die Akzeptanz bei der Bevölkerung für ihr Handeln entscheidend unterstützte.

- Daran anknüpfend führten die Befragten auch einige negative Interferenzen an: die oben genannten ,Wutbürger" hätten nach Ansicht der Change Agents enorm dazu beigetragen, dass die bayerische Landesregierung eine sehr restriktive Abstandregelung für Windkrafträder (10h) etablierte und der von den Change Agents forcierte Ausbau der Windkraft in Bayern praktisch zum Erliegen kam. Ebenfalls im Bereich der Politik, nahmen die Change Agents die fehlende klare Linie der Bundesregierung zum weiteren Verlauf der Energiewende als besonders negativ wahr: Hier fehlte nach ihrer Ansicht eine entsprechende „Umfeldreaktion“, um widersprüchliche Entwicklungstendenzen und Fehlinvestitionen zu vermeiden. Auf Bundesebene, bzw. internationaler Ebene nannten viele Change Agents die Summe aus dem Lobbyismus der großen Energiekonzerne, dem niedrigen Preis für fossile Brennstoffe sowie dem Zerfall des Elek- 
trizitätspreises als die negativsten Umfeldreaktionen, die das deutschlandweite Fortschreiten der Energiewende entscheidend verlangsamen und auch für die Change Agents vor Ort enorme Barrieren erzeugt. Durch diese nationalen und internationalen Entwicklungen fehlt den Haushalten, Firmen und Kommunen jeglicher Anreiz in weitere Maßnahmen zum Fortschritt der Energiewende zu investieren und das Engagement der Change Agents stößt aktuell kaum mehr auf positive Umfeldreaktionen in der Region.

Die jüngsten Entwicklungen, die überwiegend als negative Interferenzen wahrgenommen wurden, erzeugten bei den Change Agents eine grundlegende Frustration. Viele gaben an, aus den jüngsten Interferenzen gelernt zu haben, sich nicht mehr gleichermaßen für das Vorantreiben der regionalen Energiewende zu verausgaben und z. B. keine Informationsveranstaltungen mehr zu initiieren und auch den nationalen und bundesweiten Austausch zurückzufahren. Vielmehr konzentrierten sie sich auf ihr berufliches Engagement, das nach wie vor in Verbindung mit der Energiewende steht (z. B. Tätigkeit bei Stadtwerken, Energieagenturen oder im Bereich Gebäudesanierung). Das allgemeine Fazit der Change Agents war es, jetzt schlicht abzuwarten, bis sich die systemischen Faktoren (wie Ressourcenoder Strompreise) wieder besserten und die Energiewende wieder mit größeren Schritten voranschreite.

Rekapituliert man die exemplarischen Ergebnisse aus dieser empirischen Anwendung des HES Frameworks, hat sich gezeigt, dass es sich bei dem untersuchten Fall um ein Netzwerk von Change Agents handelt, die ähnliche, bzw. sich stark überlappende Ziele verfolgen und Strategien wählen. Ihre Handlungen sind dabei auf unterschiedlichen Ebenen lokalisiert (Weiterentwicklung einer Technologie Bau einer Anlage, Gründung einer Organisation oder Etablierung einer Politik). Sie sind dabei selbst in vielen gesellschaftlichen Bereichen verankert (u. a. Politik, Vereine, Verbände, Wirtschaft und Forschung) und verbinden diese in ihrem Akteursnetzwerk. Dieses gesellschaftlich breit vernetzte und regional gut verankerte Netzwerk konnte dann zunächst eine sehr positive Umfeldreaktion in der Region evozieren - natürlich eingebettet in die bundesweiten Entwicklungen mit Zubau der Erneuerbaren und Institutionalisierung der Energiewende. In jüngster Zeit nahmen die Change Agents jedoch viele negative Umfeldreaktionen auf unterschiedlichen Ebenen des sozialen Subsystems wahr, die zu einer Stagnation der Energiewende und persönlicher Frustration und Resignation der Change Agents führte.

\section{Diskussion und abschließende Bemerkungen}

Mit der exemplarischen empirischen Verwendung des HES Frameworks für die Fallstudie der regionalen Energiewende im Allgäu konnte gezeigt werden, dass das HES Framework mit einer Erweiterung des zugrundeliegenden Systemverständnisses gut auf sozio-technische Systeme angewandt werden kann. Es unterstützt die Analyse des individuellen Akteurshandelns in komplexen Systemen und leistet damit einen entscheidenden Beitrag dazu, die Dynamiken systemischer Transitionen besser zu verstehen. Und gerade für das Verständnis der Entwicklung und Veränderung von Menschen planvoll gestalteter technologischer Subsysteme erscheint die genauere Untersuchung der Treiber und konstituierenden Mechanismen des Akteurshandelns besonders wertvoll.

Der erweiterte Umfeldbegriff hat sich besonders in den Umfeldwahrnehmungen der Akteure bestätigt, die sie im Zusammenhang mit ihren Handlungsmotiven, Strategien und Handlungsformen anführten. Hier wurden nicht nur ökologische Motive wie der Klimawandel oder die Endlichkeit der fossilen Brennstoffe genannt, sondern z. B. auch die Sorge um das Wohl zukünftiger Generationen oder der regionalökonomischen Entwicklung des Allgäus. Der erweiterte Umfeldbegriff ermöglicht daher ein deutlich komplexeres Verständnis der Faktoren, die das Akteurshandeln in systemischen Transitionen konstituieren. Zusätzlich hat sich die explizite Berücksichtigung der Dynamiken auf unterschiedlichen Hierarchieebenen vor allem im sozialen System als sehr hilfreich erwiesen. Es konnte beispielhaft gezeigt werden, wie das Engagement der Change Agents zunächst positive Umfeldreaktionen auf allen gesellschaftlichen Ebenen erzeugte und somit von der Gruppen- auf die Ebene der Institutionen und regionalen Gesellschaft gehoben wurde. Mit fortschreitendem Institutionalisierungsprozess nahmen die Change Agents aber auch zunehmend negative Umfeldreaktionen und Interferenzen auf unterschiedlichen gesellschaftlichen Ebenen wahr. Mit der Terminologie der MLP gesprochen zeigt dies, dass die Nische an Gleichgesinnten, in der die Change Agents anfangs tätig war, mittlerweile im Regime des Energiesystems angekommen war und auf entsprechenden Widerstand stieß. Diese eingangs vorgeschlagene explizite Berücksichtigung der Interferenzen zwischen den unterschiedlichen Ebenen (hier vor allem im sozialen System) hat sich daher als sehr gewinnbringend erwiesen.

Für die weitere Konzeptualisierung dieser Interferenzen zwischen den Ebenen des sozialen Subsystems wäre ein konzeptioneller Anschluss an die MLP sehr gewinnbringend, da die MLP speziell die Analyse von Institutionalisierung und Strukturationsprozessen in Transitionen bzw. den Interferenzmechanismen zwischen unterschiedlichen sozialen Ebenen - herausarbeitet. Ein Zusammen- 
Tab. 2 Soziale Ebenen des HES Frameworks und Strukturationsebenen der MLP. (Eigene Darstellung)

\begin{tabular}{llll}
\hline HES Framework & Regulationsmechanismen & MLP & Strukturationsniveau \\
\hline Individuum, Organisation & Individuelle Normen, Werte, Ziele & Niche & $\begin{array}{l}\text { Geringe Strukturation, wenig institutionalisierte } \\
\text { Akteursnetzwerke, unabhängig von der gängigen } \\
\text { Regimelogik, schnelle Veränderung }\end{array}$ \\
Institution, Gesellschaft & $\begin{array}{l}\text { National geteilte juristische, öko- } \\
\text { nomische, politische und kulturelle }\end{array}$ & Regime & $\begin{array}{l}\text { Dominierende Regeln, Normen, Gesetze, wie z. B. } \\
\text { Politik, Märkte oder auch die Energieversorgung } \\
\text { funktioniert, langsamere Veränderung }\end{array}$ \\
& $\begin{array}{l}\text { Praktiken } \\
\text { Übernationale Werte und Visionen } \\
\text { (z. B. Menschenrechte, Klima- } \\
\text { schutzabkommen) }\end{array}$ & Landschaft & $\begin{array}{l}\text { Grundlegende Wertevorstellungen, Weltanschauun- } \\
\text { gen, langfristige Trends, sehr langsame Veränderung }\end{array}$ \\
& & \\
\hline
\end{tabular}

bringen des individualpsychologischen Hintergrunds des HES Frameworks sowie des stärker soziologisch geprägten Hintergrunds der MLP könnte eine umfassende Konzeptualisierung von Akteurshandeln in systemischen Transitionen ermöglichen und damit einen hilfreichen Beitrag zur Weiterentwicklung der Transitionsforschung leisten. Wir schlagen vor, die von Scholz (2011) etablierten Gesellschaftsebenen mit ihren entsprechenden Regulationsmechanismen den Strukturationsebenen in der MLP zuzuordnen (vgl. Tab. 2).

So könnte das HES Framework für die Transitionsforschung anschlussfähig gemacht werden und die zusätzliche Konzeption des Akteurshandelns mit Zielen, Strategien und Umfeldwahrnehmungen, die das HES-Framework bringt, in die Studien zu sozio-technischen Systemtransitionen integriert werden.

Neben der expliziteren Berücksichtigung der Rolle des Akteurshandelns und einer Struktur für dessen empirische Analyse, könnte das HES Framework z. B. auch einen Beitrag dazu leisten, die bisher als unterkonzeptualisiert geltende Landschaft weiter zu überarbeiten. Dieses höchste Strukturationsniveau könnte so z. B. aus Sicht des Individuums in interne (internalisierte) und externe Normen und Werte, bzw. gesellschaftliche Großtrends untergliedert werden. Die internalisierten Normen und Werte wären dabei beim Individuum in Form von Zielen und Motiven zu lokalisieren, die externen Normen und Werte im sozialen Umfeld, das vom Individuum wahrgenommen bzw. evaluiert wird und ebenfalls Einfluss auf das resultierende Verhalten hat. Es zeigt sich deutlich, dass ein enormes Potenzial zur gegenseitigen konzeptionellen Bereicherung zwischen der MLP bzw. der Transitionsforschung und dem HES Framework bzw. den Mensch-Umwelt Studien vorhanden ist.

Einschränkend müssen jedoch folgende Limitationen festgehalten werden: unsere Anwendung und die daraus abgeleiteten Implikationen basieren lediglich auf einer exemplarischen Fallstudie, die explorativ und rein auf die Wahrnehmung der Change Agents fokussiert war. Das Berücksichtigen anderer Umfeldwahrnehmungen (z.B. von Politikern, Unternehmern und der regionalen Bevölkerung) könnte hier eine methodische Ergänzung sein. Weiterhin ist die Verschränkung qualitativer und quantitativer Methoden zur Systemanalyse aber auch zur Erhebung der Umfeldwahrnehmung eine wichtige Ergänzung. Zur verbesserten Analyse der Kausalitäten zwischen Umfeldwahrnehmungen und Handlungen, könnten mit den Befragten gemeinsam z. B. Causal Loop Diagramme erarbeitet werden. Sie bieten eine einfache und transdisziplinär nutzbare methodische Grundlage, sich auf die wichtigsten Wahrnehmungen und ihre kausale Relation zu den Handlungen zu konzentrieren. Abschließend möchten wir auch noch einmal betonen, dass das HES Framework vor allem für die Analyse individuellen Akteurshandelns entwickelt wurde und hierin seine größte Stärke liegt. Eine Anwendung für Gruppen und Organisationen müsste anhand weiterer empirischer Arbeit kritisch geprüft werden.

Weiterer Forschungsbedarf besteht unserer Ansicht nach zum einen in der Anwendung des Frameworks auf andere Sektoren (z. B. Mobilität oder Ernährung) und einer genaueren Untersuchung der Feedbacks und Interferenzen zwischen den verschiedenen Subsystemen aber auch innerhalb der Subsysteme zwischen den Ebenen. Zum anderen kann im weiteren Kontext der Transitionsforschung die empirische Anwendung des HES-Frameworks auch zur Analyse der Transitionsphasen über die Zeit angewandt werden, um zu verstehen, wie aus dem Wechselspiel zwischen Umfeldwahrnehmung, Akteurshandeln, Umfeldreaktion und deren Evaluation über längere Zeiträume bestimmte Transitionspfade entstehen. In diesem Zusammenhang könnte analysiert werden, inwiefern die Motive, Strategien und daraus resultierenden Handlungsweisen der Change Agents z. B. „regimekonform“ sind oder nicht und welchen Einfluss dies auf die Geschwindigkeit und den Erfolg der Transition hat. Weiterhin könnte somit auch dem Lernen von Akteuren ein größeres konzeptionelles Gewicht gegeben werden. Es könnte beispielsweise stärker berücksichtigt werden wie sich Motive, Umfeldwahrnehmungen und Handlungen von Change Agents über die Zeit verändern aber auch wie diese Veränderungen in das systemische Umfeld eingebettet sind - zum Beispiel welchen Einfluss soziale Netzwerke auf die individuellen Wahrnehmungen und Verhaltensweisen ausüben. Damit würde die Rolle von individuellem und sozia- 
lem Lernen in systemischen Transitionsprozessen stärkere Berücksichtigung finden.

Schließlich sollte in der Transitionsforschung weiter an der Erarbeitung eines Frameworks für sozio-technische Transition gearbeitet werden, dass sowohl systemische Transitionsmechanismen, wie z.B. die Rolle von Strukturationsprozessen berücksichtigt als auch das genaue Verständnis der Rolle des Akteurshandelns, seiner konstituierenden Faktoren sowie seiner Einbettung und Interaktion in und mit dem systemischen Umfeld. Damit würde der Forderung nach der Einbettung von agency nachgekommen und ein entscheidender Beitrag zur Dynamisierung der Transitionsframeworks geleistet. Der Vorschlag des Anschlusses des HES Frameworks an die MLP stellt einen ersten Schritt in diese Richtung dar.

Danksagung Die Autoren bedanken sich bei Holger Sauter und Thomas Knöpfle für die Unterstützung bei der Datenerhebung sowie allen Interviewpartnern in der Region, die sich zusätzlich zu ihrem Engagement die Zeit für die Interviews genommen haben.

Förderung Wir danken der Swiss Mobiliar für die Co-Finanzierung dieser Arbeit.

Open Access Dieser Artikel wird unter der Creative Commons Namensnennung 4.0 International Lizenz (http://creativecommons.org/ licenses/by/4.0/deed.de) veröffentlicht, welche die Nutzung, Vervielfältigung, Bearbeitung, Verbreitung und Wiedergabe in jeglichem Medium und Format erlaubt, sofern Sie den/die ursprünglichen Autor(en) und die Quelle ordnungsgemäß nennen, einen Link zur Creative Commons Lizenz beifügen und angeben, ob Änderungen vorgenommen wurden.

\section{Literatur}

Avelino F, Rotmans J (2009) Power in transition: an interdisciplinary framework to study power in relation to structural change. Eur J Soc Theory 12(4):543-569. doi:10.1177/1368431009349830

Avelino F, Wittmayer J, Haxeltine A, Kemp R, O'Riordan T, Weaver P, Loorbach D, Rotmans J (2014) Game changers and transformative social innovation. The case of the economic crisis and the new economy. http://www.transitsocialinnovation.eu/content/ original/Book\%20covers/Local\%20PDFs/178\%20TRANSIT_ WorkingPaper1_Gamechangers_Avelino.pdf (24S). Zugegriffen: 16.06.2017

Avelino F, Wittmayer JM (2016) Shifting power relations in sustainability transitions: a multi-actor perspective. J Environ Policy Plan 18(5):628-649. doi:10.1080/1523908x.2015.1112259

Binder CR, Hecher M, Vilsmaier U (2014) Visionen, Institutionen und Infrastrukturen als Elemente der Energietransformation. In: Klima von unten: Regionale Governance und gesellschaftlicher Wandel. Campus, Frankfurt a. M., S 267-286

Binder CR, Hinkel J, Bots PWG, Pahl-Wostl C (2013) Comparison of frameworks for analyzing social-ecological systems. Ecol Soc 18(4):26

de Haan JH, Rotmans J (2011) Patterns in transitions: understanding complex chains of change. Technol Forecast Soc Change 78(1):90-102 (Abgerufen von http://www.sciencedirect.com/ science/article/pii/S0040162510002234)
Deutsche Gesellschaft für Sonnenenergie e. V. (o.J.): EnergyMap - Energieregionen - Schwaben. http://www.energymap.info/ energieregionen/DE/105/111/169.html. Zugegriffen: 16.06.2017

Fischer L-B, Newig J (2016) Importance of actors and agency in sustainability transitions: a systematic exploration of the literature. Sustainability 8(5):476 (Abgerufen von http://www.mdpi.com/ 2071-1050/8/5/476/htm)

Geels FW (2002) Technological transitions as evolutionary reconfiguration processes: a multi-level perspective and a case-study. Res Policy 31(8-9):1257-1274. doi:10.1016/s0048-7333(02)00062-8

Geels FW (2004) From sectoral systems of innovation to sociotechnical systems: insights about dynamics and change from sociology and institutional theory. Res Policy 33(6):897-920 (Abgerufen von http://www.sciencedirect.com/science/article/pii/ S0048733304000496)

Geels FW (2011) The multi-level perspective on sustainability transitions: responses to seven criticisms. Environ Innov Soc Transit 1(1):24-40. doi:10.1016/j.eist.2011.02.002

Geels FW, Kemp R (2006) Transitions, transformations and reproduction: dynamics in socio-technical systems. Oxford University Press, Oxford (Abgerufen von http://www.druid.dk/uploads/tx_ picturedb/ds2005-1462.pdf)

Geels FW, Schot J (2007) Typology of sociotechnical transition pathways. Res Policy 36(3):399-417. doi:10.1016/j.respol.2007.01. 003

Genus A, Coles A-M (2008) Rethinking the multi-level perspective of technological transitions. Res Policy 37(9):1436-1445

Giddens A (1984) The constitution of society: outline of the theory of structuration. University of California Press, Berkley

Grin J, Rotmans J, Schot J (2011) On patterns and agency in transition dynamics: some key insights from the KSI programme. Environ Innov Soc Transit 1(1):76-81 (Abgerufen von http://www. sciencedirect.com/science/article/pii/S2210422411000190)

Hecher M, Vilsmaier U, Akhavan R, Binder CR (2016) An integrative analysis of energy transitions in energy regions: a case study of ökoEnergieland in Austria. Ecol Econ 121:40-53

Heins B, Alscher S (2013) Change Agents-,,Pioniere des Wandels "als Akteure für Klimaschutz und Energiewende. In: Klimaschutz \& Energienachhaltigkeit: Die Energiewende als sozialwissenschaftliche Herausforderung. universaar, Saarbrücken, S 119-134

Loorbach D, Van Der Brugge R, Taanman M (2008) Governance in the energy transition: practice of transition management in the Netherlands. Int J Environ Technol Manag 9(2-3):294-315. doi:10. 1504/ijetm.2008.019039

Mayring P (2010) Qualitative Inhaltsanalyse. In: Handbuch qualitative Forschung in der Psychologie. Springer, Heidelberg, S 601-613

Mühlemeier S, Knöpfle T (2016) „Pioniere der Energiewende“ Bevölkerungsbefragung im baerischen Allgäu. For Change Working Paper.

Mühlemeier S, Wyss R, Binder CR (2017) Ein indikatorengestützter Ansatz zur Analyse der Resilienz in Transitionen von Energiesystemen: theoretische Konzeptualisierung und empirische Anwendung im bayerischen Allgäu. In: Resilienz. Springer

Neffke F, Hartog M, Boschma R, Henning M et al (2014) Agents of structural change. The role of firms and entrepreneurs in regional diversification. Utrecht University, Section of Economic Geography, Utrecht (Abgerufen von https://ideas.repec.org/p/egu/ wpaper/1410.html)

Riddell D, Tjornbo O, Westley F (2012) Agency and innovation in a phase of turbulent change: conservation in the Great Bear Rainforest. In: Using a positive lens to explore social change and organizations. Routledge, New York, S 155-180 (Abgerufen von https://books.google.ch/books?hl=de\&lr=\&id=yBmmQYL99gC\&oi=fnd\&pg=PA155\&dq=Riddell, +D., + Tj\%C3 $\%$ B6rnbo, + O., +Westley, + F.+(2012):+Agency+and+innovation+ in $+\mathrm{a}+$ phase+of+turbulent+change:+conservation+in+the+Great+ Bear+Rainforest.+In:+Golden-Biddle, + K.+\%26+Dutton, + J.+E. $+($ editors):+Using+a+positive+lens+to+ex-plore+social+change 
+ and+organizatio\&ots=zHVNQ134XC\&sig=7DS_zgda_LKCID Tgm1GLjFQcoxA). Zugegriffen: 16.06.2017

Rotmans J, Loorbach D (2009) Complexity and transition management. J Ind Ecol 13(2):184-196. doi:10.1111/j.1530-9290.2009. 00116.x

Scholz R (2011) The HES postulates. In: Environmental literacy in science and society from knowledge to decisions. Cambridge University Press, Cambridge, S 407-452

Scholz RW (2011) Environmental literacy in science and society: from knowledge to decisions. Cambridge University Press, Cambridge

Scholz RW, Binder CR (2003) The paradigm of human-environment systems. http://e-collection.library.ethz.ch/eserv/eth:26365/eth26365-01.pdf. Zugegriffen: 16.06.2017

Scholz RW, Binder CR (2004) Principles of human-environment systems (HES) research (thesis). http://iemss.logismi.co:8080/xmlui/ handle/iemss/4011 (Erstellt: 21. Sept 2004) (International Environmental Modelling and Software Society)

Scholz RW, Binder CR (2011) The HES framework. In: Environmental literacy in science and society: from knowledge to decisions. Cambridge University Press, Cambridge, S 453-462

Scholz RW, Binder CR, Lang DJ (2011) The HES framework. In: Environmental literacy in science and society: from knowledge to decisions. Cambridge University Press, Cambridge
Smith A, Stirling A, Berkhout F (2005) The governance of sustainable socio-technical transitions. Res Policy 34(10):1491-1510. doi:10. 1016/j.respol.2005.07.005

Sommer B, Schad M (2014) Change agents for climate change mitigation in urban areas. GAIA 23(1):48-54 (Abgerufen von http:// www.ingentaconnect.com/content/oekom/gaia/2014/00000023/ 00000001/art00011)

Späth P, Koblmüller M, Kubeczko K, Faber F, Bärnthaler J, Bergmann $\mathrm{H}$, Breisler A et al (2007) Energieregionen: Wirksame Leitbildprozesse und Netzwerke zur regionalen Gestaltung sozio-technischen Wandels. Berichte aus Energie-und Umweltforschung, Bd. 29. BMVIT, Vienna (Abgerufen von https://www.klimafonds. gv.at/assets/Uploads/Blue-Globe-Reports/Forschung/2008-2010/ BGR52010KB07EZ2F44369FSER-Net.pdf)

Westley FR, Tjornbo O, Schultz L, Olsson P, Folke C, Crona B, Bodin Ö (2013) A theory of transformative agency in linked socialecological systems. Ecol Soc 18(3):27 (Abgerufen von https://dlc. dlib.indiana.edu/dlc/handle/10535/9134)

Wittmayer J (2016) Transition management, action research and actor roles: understanding local sustainability transitions. https://repub. eur.nl/pub/94385/. Zugegriffen: 17.06.2017

Wittmayer JM, Schäpke N (2014) Action, research and participation: roles of researchers in sustainability transitions. Sustain Sci 9(4):483-496. doi:10.1007/s11625-014-0258-4 